\title{
Correlation between patient health questionnaire-2 and postoperative pain in laparoscopic cholecystectomy
}

\section{Yusom Shin, Tae Woo Park, Huiyoung Kim, Dong-jin Shim, Hochul Lee, Joo-Duck Kim, and Donghee Kang}

Department of Anesthesiology and Pain Medicine, Kosin University College of Medicine, Busan, Korea
Received July 24, 2019

Revised September 25, 2019

Accepted October 23, 2019

\section{Corresponding author}

Donghee Kang, M.D.

Department of Anesthesiology and

Pain Medicine, Kosin University

College of Medicine, 262 Gamcheon-

ro, Seo-gu, Busan 49267, Korea

Tel: 82-51-990-6281

Fax: 82-51-254-2504

E-mail: lorien@kosin.ac.kr
Background: Postoperative pain is affected by preoperative depression. If the risk of postoperative pain associated with depression can be predicted preoperatively, anesthesiologists and/or surgeons can better manage it with personalized care. The objective of this study was to determine the efficacy of Patient Health Questionnaire-2 (PHQ-2) depression screening tool as a predictor of postoperative pain.

Methods: A total of 50 patients scheduled for elective laparoscopic cholecystectomy with an American Society of Anesthesiologists physical status 1 or 2 were enrolled. They answered the PHQ-2, which consists of two questions, under the supervision of a researcher on the day before the surgery. The numerical rating scale (NRS) scores were assessed at post-anesthesia care unit (PACU), at 24 , and 48 postoperative hours, and the amount of intravenous patient-controlled analgesia (IV-PCA) administered was documented at 24, 48, and 72 postoperative hours. At $72 \mathrm{~h}$, the IV-PCA device was removed and the final dosage was recorded.

Results: The NRS score in PACU was not significantly associated with the PHQ-2 score (correlation coefficients: $0.13[P=0.367])$. However, the use of analgesics after surgery was higher in patients with PHQ-2 score of 3 or more (correlation coefficients: 0.33 [P=0.018]).

Conclusions: We observed a correlation between the PHQ-2 score and postoperative pain. Therefore, PHQ-2 could be useful as a screening test for preoperative depression. Particularly, when 3 points were used as the cut-off score, the PHQ-2 score was associated with the dosage of analgesics, and the analgesic demand could be expected to be high with higher PHQ-2 scores.

Keywords: Analgesics; Depression; Patient health questionnaire; Postoperative pain.

\section{INTRODUCTION}

Postoperative pain causes patients discomfort and vasoconstriction through sympathetic hyperactivity, which may decelerate wound healing and increase the infection rate $[1,2]$. Therefore, appropriate pain control is important. Proper postoperative pain control reduces morbidity and shortens hospitalization and convalescence [3]. Several factors influence postoperative pain, but depression may significantly heighten its intensity $[4,5]$.

The prevalence of depression is increasing in most countries, including Korea, where the lifetime and annual prevalence are $6.7 \%$ and $3.0 \%$ [6]. To screen patients with depression, many measures, such as the Patient Health Ques-

This is an Open Access article distributed under the terms of the Creative Commons Attribution Non-Commercial License (http://creativecommons.org/licenses/by-nc/4.0) which permits unrestricted non-commercial use, distribution, and reproduction in any medium, provided the original work is properly cited.

Copyright (C) the Korean Society of Anesthesiologists, 2020 
tionnaire-9 (PHQ-9) [7], Center for Epidemiological Studies Depression Scale [8], Beck Depression Inventory [9], and Geriatric Depression Scale [10], have been developed. However, $30-50 \%$ of depressed patients are misdiagnosed due to difficulties in diagnosis $[11,12]$.

Postoperative pain is associated with depression, therefore screening for preoperative depression might help with better management of postoperative pain [4,5]. However, screening for depression in pre-anesthetic assessments is difficult, as it is time-consuming. Moreover, depression screening is difficult for anesthesiologists, as it generally requires professional knowledge. To screen for depression in pre-anesthetic assessments, the screening test should be short, with high reliability and validity. Therefore, PHQ-2 could be an appropriate option, since it evaluates patients using only two of the nine questions of PHQ-9, which is the conventional depression screening test [13].

In this study, we evaluated the usefulness of the PHQ-2 test for preoperative depression evaluation. If there was a correlation between preoperative PHQ-2 and postoperative pain, we considered that PHQ-2 could be used to predict postoperative pain without a definite diagnosis of depression.

\section{MATERIALS AND METHODS}

This study was approved by our committee for Clinical Research Ethics (no. KUGH 2017-08-033-006). A total of 55 patients participated in this study. The correlation coefficient of the study was determined to be 0.4 , which can be interpreted as a fair result between 0.4 and 0.6 , referring to the previous study [14]. The minimum number of samples was 47 when $=0.05, \quad=0.20$, and correlation coefficient is 0.4 , and the study was conducted in 55 patients considering the losers. Patients identified as American Society of Anesthesiologist physical status class I-II were scheduled for laparoscopic cholecystectomy under general anesthesia. Patients with a history of depression, current medication, or complaint of post-anesthetic nausea and vomiting in previous surgeries were excluded from this study.

An anesthesiologist, who did not have prior information regarding the patient, visited the ward to examine the PHQ-2 scores of the patients who were hospitalized a day before the operation. Subsequently, he explained the use of intravenous patient-controlled analgesia (IV-PCA). There were two types of PHQ-2: Short-answer PHQ-2 and score-type PHQ-2. In this study, score-type PHQ-2 was used. The PHQ-2 questionnaire consisted of two items: "Little interest or pleasure in doing things" and "Feeling down, depressed, or hopeless." The available answers were: "Not at all," "Several days," "More than half the days," and "Nearly every day." Each question was scored on a scale of $0-3$, and the scores from both questions were added. The patient's medical records (age, height, weight, and history of psychological disorder) were also obtained.

Pre-anesthetic medication was not administered. Propofol $(1 \mathrm{mg} / \mathrm{kg})$ and remifentanil $(1 \mu \mathrm{g} / \mathrm{kg})$ were used to induce anesthesia. Anesthesia was maintained using sevoflurane and remifentanil, and remifentanil was discontinued during extubation. In the recovery room, fentanyl $50 \mu \mathrm{g}$ was administered in the loading dose and IV-PCA was connected. The patient was then told to press the button of the IV-PCA directly if there was pain. An IV-PCA device was used with Hospira Gemstar Blue (GemStar ${ }^{\mathrm{TM}}$, Hospira Inc., USA). We mixed 1,000 $\mu$ g of fentanyl (fentanyl citrate, Hana Pharm, Korea), 50 mg of nefopam (nefopam HCl, Ilsung Pharm, Korea), and $4 \mathrm{mg}$ of ondansetron to obtain a total volume of $150 \mathrm{ml}$. When the bolus button was pressed, 1.5 $\mathrm{ml}(10 \mu \mathrm{g}$ of fentanyl) of the drug mixture was injected. The lock time was $5 \mathrm{~min}$.

The degree of postoperative pain on a numerical rating scale (NRS) and the usage amount of IV-PCA were assessed. The NRS scores were assessed at PACU, 24, and 48 postoperative hours. Usage amount of IV-PCA was collected at 24,48 , and 72 postoperative hours. At $72 \mathrm{~h}$, the IVPCA device was removed. When a patient complained of nausea or vomited while using IV-PCA, $4 \mathrm{mg}$ of ondansetron was additionally administered. When the complaint sustained, IV-PCA was discontinued.

A statistical analysis was performed using the SPSS (version 24.0, IBM, USA) statistical program. To analyze the correlations among the PHQ-2 score, NRS score, and fentanyl use, point-biserial correlation or Pearson correlation analyses were used. Quantitative variables were expressed as mean $\pm \mathrm{SD}$, and $\mathrm{P}<0.05$ was the significance level of the test.

\section{RESULTS}

A total of 55 patients who underwent laparoscopic cholecystectomy under general anesthesia were enrolled in this study, of which 8 complained of postoperative nausea and received $4 \mathrm{mg}$ of ondansetron intravenously. Out of the 8 patients, 5 complained of nausea and discontinued IV-PCA 


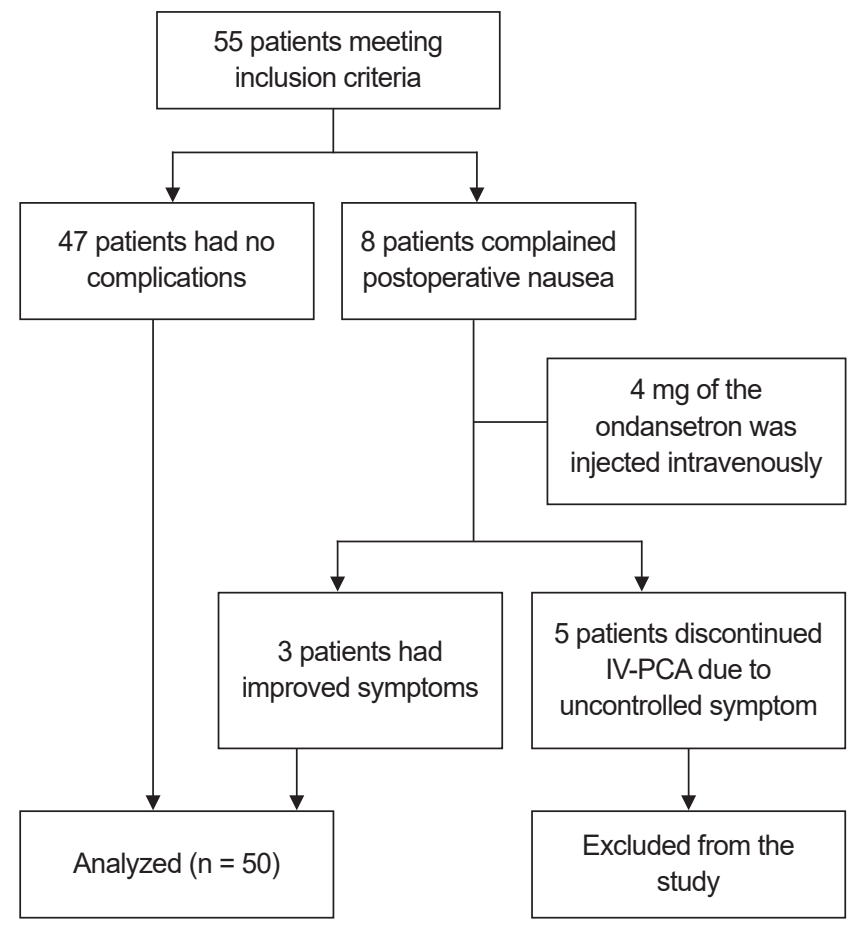

Fig. 1. A study flow chart. IV-PCA: intravenous patient-controlled analgesia.

Table 2. Coefficient of Point-biserial Correlation or Pearson Correlations between Demographic Data/PHQ-2 Score and NRS Score/Fentanyl Consumption

\begin{tabular}{lcccc}
\hline \multirow{2}{*}{ Variable } & \multirow{2}{*}{$\begin{array}{c}\text { NRS score } \\
\text { in PACU }\end{array}$} & \multicolumn{3}{c}{ Fentanyl consumption } \\
\cline { 3 - 5 } & & POD1 & POD2 & POD3 \\
\hline Sex (M/F) & -0.25 & & & -0.69 \\
Age (yr) & -0.12 & & & $-0.34^{*}$ \\
PHQ-2 score & 0.13 & $0.68^{\dagger}$ & $0.45^{*}$ & 0.24 \\
\hline
\end{tabular}

PHQ-2: Patient Health Questionnaire-2, NRS: numerical rating scale, POD: post-operative day, PACU: post-anesthesia care unit. POD3 means total fentanyl consumption. ${ }^{*} \mathrm{P}<0.05,{ }^{\dagger} \mathrm{P}<0.001$.

due to uncontrolled symptoms after a single administration of $4 \mathrm{mg}$ of ondansetron and were excluded from the study. Finally, we analyzed 50 patients (Fig. 1). Table 1 shows the demographic characteristics. Side effects other than nausea did not occur. In this study, the PHQ-2 was conducted for about two minutes, and no patient refused the survey.

Postoperative NRS in recovery room and total fentanyl usage were not significantly associated with the patient's PHQ-2 score (correlation coefficients: $0.13[\mathrm{P}=0.367]$ and 0.24 [P = 0.098], Table 2). When three points on PHQ-2 was used as the cut-off score, the fentanyl use was significantly higher in patients who scored $\geq 3$ points than in those
Table 1. Characteristics of Patients

\begin{tabular}{lc}
\hline \multicolumn{1}{c}{ Variable } & Value \\
\hline Sex $(\mathrm{M} / \mathrm{F})$ & $28 / 22$ \\
Age $(\mathrm{yr})$ & $50.9 \pm 10.8$ \\
Weight $(\mathrm{kg})$ & $66.3 \pm 13.6$ \\
ASA physical status I/II & $1938-12-01$ \\
Preoperative PHQ-2 score & \\
0 & $24(48)$ \\
1 & $10(20)$ \\
2 & $5(10)$ \\
3 & $6(12)$ \\
4 & $5(10)$ \\
5 & $0(0)$ \\
6 & $0(0)$ \\
Remifentanil total dose $(\mu \mathrm{gg})^{*}$ & $450.9 \pm 57.1$ \\
Fentanyl total dose $(\mu \mathrm{g})^{\dagger}$ & \\
PHQ-2 score $\geq 3$ & $371.6 \pm 71.5$ \\
PHQ-2 score $<3$ & $300.3 \pm 88.6$ \\
Duration of anesthesia $(\mathrm{min})$ & $63.3 \pm 13.7$
\end{tabular}

Values are presented as number (\%) or mean \pm SD. ASA: American Society of Anesthesiologists, PHQ-2: Patient Health Questionnaire-2, IV-PCA: intravenous patient-controlled analgesia. *Total amount of remifentanil during surgery. ${ }^{\dagger}$ Total amount of fentanyl in IV-PCA. ${ }^{\dagger}$ Total amount of fentanyl in IV-PCA.

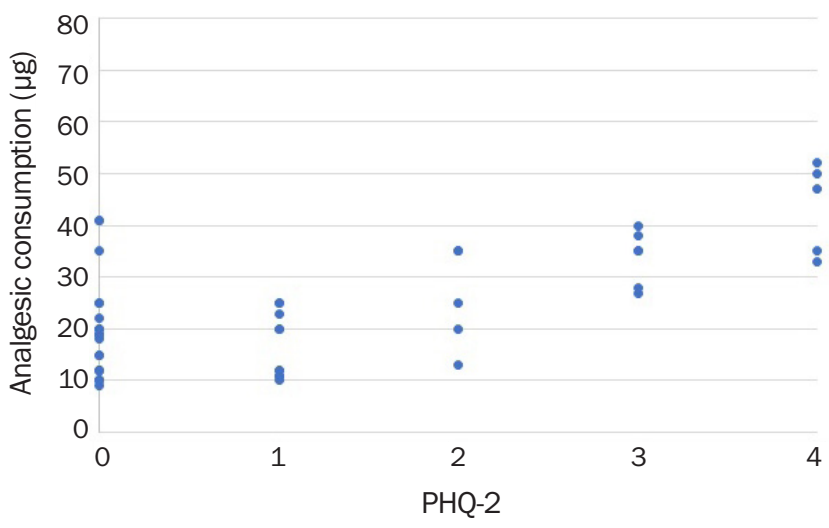

Fig. 2. Relationship between preoperative Patient Health Questionnaire-2 (PHQ-2) score and analgesic consumption on the first day after surgery (correlation coefficient $r=0.68[P<0.001]$ ).

scoring < 3 points ( $371.6 \pm 71.5$ vs. $300.3 \pm 88.6)$, and total fentanyl usage correlated with the PHQ-2 score (correlation coefficients: $0.33[\mathrm{P}=0.018])$. Fentanyl usage at 24 and 48 postoperative hours were significantly associated with the PHQ-2 score (correlation coefficients: 0.68 [P < $0.001]$ and $0.45[\mathrm{P}=0.010]$, Table 2). Figs. 2 , 3, and 4 show the relationship between PHQ-2 score and analgesic consumption on first, second, and third day after surgery. Age was associated with total fentanyl usage but sex was not (correlation coefficients: $-0.34[\mathrm{P}=0.016]$ and $-0.69[\mathrm{P}=$ 


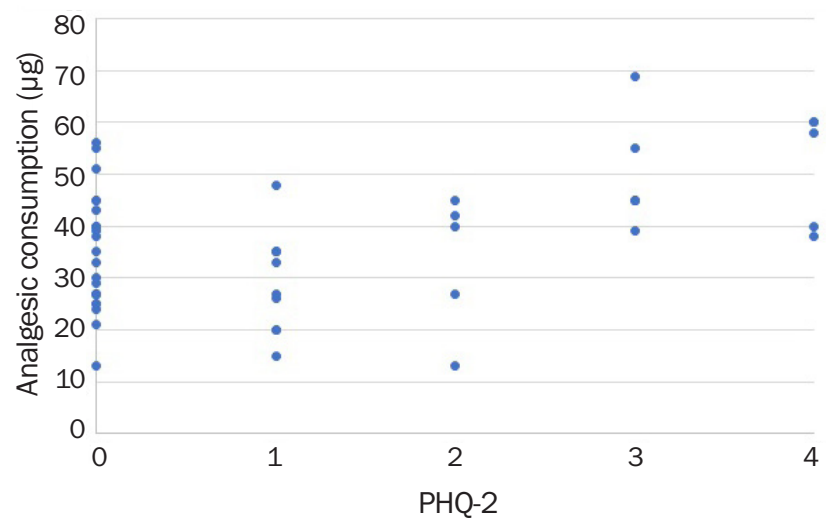

Fig. 3. Relationship between preoperative Patient Health Questionnaire-2 (PHQ-2) score and analgesic consumption on the second day after surgery (correlation coefficient $r=0.45[P=$ 0.010]).

0.640], Table 2).

\section{DISCUSSION}

The purpose of this study was to evaluate the correlation between preoperative PHQ-2 score and postoperative pain. PHQ-2 is a simple screening test using two of the nine questions of PHQ-9, a previously developed depression screening test [13]. The two questions addressed depressed mood and anorexia. There is a significant correlation with other screening tests, and PHQ-2 is known to increase the total score depending on the severity of depression in the patient group [15].

In this study, the preoperative PHQ-2 scores did not significantly correlate with the postoperative NRS scores measured in the PACU or the total fentanyl usage (correlation coefficients: 0.13 [P = 0.367] and 0.24 [P = 0.098]). However, fentanyl usage during 24 postoperative hours highly correlated with the preoperative PHQ-2 scores $(0.68[\mathrm{P}<$ $0.001]$ ). Various studies on the association between postoperative pain and preoperative depression have been conducted. De Cosmo et al. [14] reported that depression was associated with tramadol use in patients after laparoscopic cholecystectomy. Schade et al. [16] reported that preoperative depression affects postoperative pain and rehabilitation in patients with lumbar disc herniation. Oztekin et al. [17] reported that anterior cruciate ligament surgery was not associated with postoperative pain despite high preoperative depression scores in the patients. Kavakci et al. [18] found that preoperative anxiety has a greater impact on postoperative pain compared to depression in

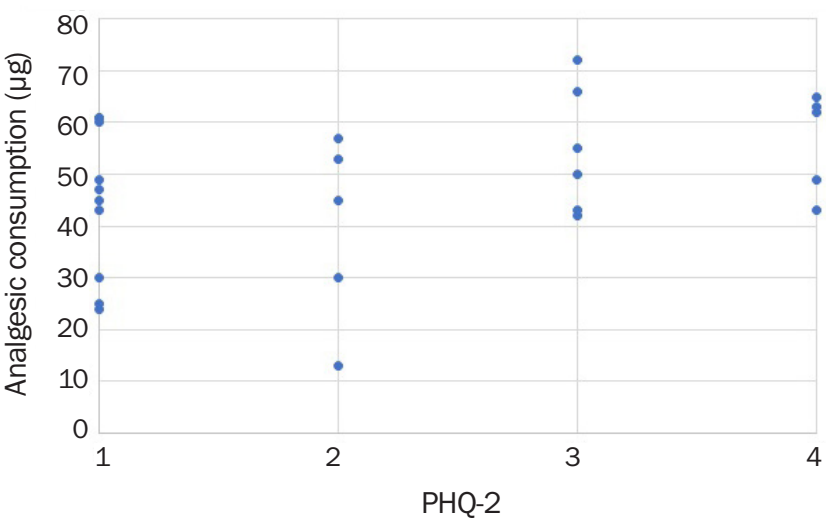

Fig. 4. Relationship between preoperative Patient Health Questionnaire-2 (PHQ-2) score and analgesic consumption on the third day after surgery (correlation coefficient $r=0.24[P=0.098]$ ).

cases of ear, nose, and neck surgery. These differences are probably because of differences in the type of surgery and type of postoperative analgesics used.

Patients who were included in this study underwent laparoscopic cholecystectomy. The surgery was less painful compared to open cholecystectomy, and IV-PCA with fentanyl controlled postoperative pain appropriately [19]. Immediately after the surgery, patients with higher PHQ-2 score had higher analgesic requirements. However, as the postoperative pain was controlled with a strong opioid, the appropriate pain control effect might have been achieved regardless of the PHQ-2 score, and it is believed that there was no difference in usage.

PHQ-2 scores did not significantly correlate with total fentanyl usage. However, after selecting the appropriate cut-off score for PHQ-2, the PHQ-2 scores were associated with total analgesic requirement. The sensitivity and specificity of depression were $91.9 \%$ and $100 \%$, respectively, when the cut-off score on PHQ-2 was 3 [15]. In this study, when 3 points were used as cut-off score, fentanyl use was significantly higher in patients who scored 3 or more points. Therefore, 3 points as a cut-off score on PHQ-2 may be appropriate for preoperative depression screening.

In this study, sex did not affect analgesic use (correlation coefficient: $-0.69[\mathrm{P}=0.640])$. In another study on analyzed factors affecting postoperative pain, sex did not affect the postoperative pain or analgesic use [4]. However, age was a major predictor of postoperative pain and analgesic use [4]. In this study, the use of analgesics was higher in younger patients (correlation coefficient: $-0.34[\mathrm{P}=$ $0.016])$. 
This study has a few limitations. First, anxiety was not investigated. Anxiety plays a major role in acute pain, including postoperative pain [18]. Brander et al. [20] reported that depression had a greater effect on postoperative chronic pain than anxiety. Patients' anxiety before surgery should have been examined to confirm its association with pain. Second, the diagnosis of depression was not an inclusion criterion. Therefore, in a controlled study on patients who were diagnosed or treated for depressive disorders, we intend to assess the efficacy of PHQ-2 as a predictor of postoperative pain. Marazziti et al. [21] reported that in the group with depression, the pain threshold was lower than in the group without depression. Depressed patients who had lower thresholds for postoperative pain were believed to have higher analgesic requirements and more negative effects of pain. Future studies could evaluate pain control or patient satisfaction when controlling postoperative pain more aggressively by increasing the amount of pain medication IV-PCA in patients with high PHQ-2 scores.

In conclusion, the PHQ-2 test is composed of two simple questions and can be performed in two minutes; therefore, it could be useful as a screening test for preoperative depression. Despite the simple questions, it highly correlated with fentanyl consumption in the early postoperative period. Particularly, when 3 points were used as the cut-off score, the PHQ-2 score was associated with the dosage of analgesics, and the analgesic demand could be expected to be high with higher PHQ-2 scores.

\section{CONFLICTS OF INTEREST}

No potential conflict of interest relevant to this article was reported.

\section{AUTHOR CONTRIBUTIONS}

Conceptualization: Yusom Shin. Data acquisition: Tae Woo Park, Dong-jin Shim. Formal analysis: Huiyoung Kim. Supervision: Joo-Duck Kim, Donghee Kang. Writing-original draft: Yusom Shin, Tae Woo Park. Writing-review \& editing: Hochul Lee, Donghee Kang.

\section{ORCID}

Yusom Shin, https://orcid.org/0000-0002-4829-0866

Tae Woo Park, https://orcid.org/0000-0003-4032-4424

Huiyoung Kim, https://orcid.org/0000-0003-2330-1458
Dong-jin Shim, https://orcid.org/0000-0002-8704-529X

Hochul Lee, https://orcid.org/0000-0002-7740-9424

Joo-Duck Kim, https://orcid.org/0000-0002-9236-5183

Donghee Kang, https://orcid.org/0000-0001-6614-9244

\section{REFERENCES}

1. Kiecolt-Glaser JK, Page GG, Marucha PT, MacCallum RC, Glaser R. Psychological influences on surgical recovery. Perspectives from psychoneuroimmunology. Am Psychol 1998; 53: 1209-18.

2. Derbyshire DR, Smith G. Sympathoadrenal responses to anaesthesia and surgery. Br J Anaesth 1984; 56: 725-39.

3. Kehlet H, Holte K. Effect of postoperative analgesia on surgical outcome. Br J Anaesth 2001; 87: 62-72.

4. Ip HYV, Abrishami A, Peng PW, Wong J, Chung F. Predictors of postoperative pain and analgesic consumption: a qualitative systematic review. Anesthesiology 2009; 111: 657-77.

5. Vaughn F, Wichowski H, Bosworth G. Does preoperative anxiety level predict postoperative pain? AORN J 2007; 85: 589-604.

6. Ahn J. Depression, suicide, and Korean society. J Korean Med Assoc 2012; 55: 320-1.

7. Spitzer RL, Kroenke K, Williams JBW; Patient Health Questionnaire Primary Care Group. Validation and utility of a self-report version of PRIME-MD: the PHQ primary care study. JAMA 1999; 282: 1737-44.

8. Radloff LS. The CES-D scale: a self-report depression scale for research in the general population. Appl Psychol Meas 1977; 1: 385-401.

9. Beck AT, Guth D, Steer RA, Ball R. Screening for major depression disorders in medical inpatients with the Beck Depression Inventory for Primary Care. Behav Res Ther 1997; 35: 785-91.

10. Yesavage JA, Brink TL, Rose TL, Lum O, Huang V, Adey M, et al. Development and validation of a geriatric depression screening scale: a preliminary report. J Psychiatr Res 1982; 17: 37-49.

11. Gerber PD, Barrett J, Barrett J, Manheimer E, Whiting R, Smith R. Recognition of depression by internists in primary care: a comparison of internist and "gold standard" psychiatric assessments. J Gen Intern Med 1989; 4: 7-13.

12. Susman JL, Crabtree BF, Essink G. Depression in rural family practice: easy to recognize, difficult to diagnose. Arch Fam Med 1995; 4: 427-31.

13. Tuck AN, Scribani MB, Grainger SD, Johns CA, Knight RQ. The 9-Item Patient Health Questionnaire (PHQ-9): an aid to assessment of patient-reported functional outcomes after spinal surgery. Spine J 2018; 18: 1398-405.

14. De Cosmo G, Congedo E, Lai C, Primieri P, Dottarelli A, Aceto P. Preoperative psychologic and demographic predictors of pain perception and tramadol consumption using intravenous pa- 
tient-controlled analgesia. Clin J Pain 2008; 24: 399-405.

15. Shin JH, Kim HC, Jung CH, Kim JB, Jung SW, Cho HJ, et al. The Standardization of the Korean Version of the Patient Health Questionnaire-2. J Korean Neuropsychiatr Assoc 2013; 52: 11521.

16. Schade V, Semmer N, Main CJ, Hora J, Boos N. The impact of clinical, morphological, psychosocial and work-related factors on the outcome of lumbar discectomy. Pain 1999; 80: 239-49.

17. Oztekin HH, Boya H, Ozcan O, Zeren B, Pinar P. Pain and affective distress before and after ACL surgery: a comparison of amateur and professional male soccer players in the early postoperative period. Knee 2008; 15: 368-72.

18. Kavakci Ö, Altuntas EE, Müderris S, Kugu N. Effects of the pre- operative anxiety and depression on the postoperative pain in ear, nose and throat surgery. Indian J Otol 2012; 18: 82-7.

19. Kang DH, Kim DS, Kim JD, Kim JW. A comparison of fentanyl and morphine for patient controlled analgesia after laparoscopic cholecystectomy. Anesth Pain Med 2013; 8: 21-5.

20. Brander VA, Stulberg SD, Adams AD, Harden RN, Bruehl S, Stanos SP, et al. Predicting total knee replacement pain: a prospective, observational study. Clin Orthop Relat Res 2003; (416): 27-36.

21. Marazziti D, Castrogiovanni P, Rossi A, Rosa C, Ghione S, Di Muro A, et al. Pain threshold is reduced in depression. Int J Neuropsychopharmacol 1998; 1: 45-8. 